\title{
Der Allergologe - ein fachübergreifender Spezialist?
}

$\mathrm{n}$ einer Zeit, in der man mit wissenschaftlichen Daten und Problemen des klinischen Alltags exponenziell überflutet wird, ist es nicht immer einfach, den eigenen Stellenwert klar zu definieren. So fragt man sich, wie die Volkskrankheit „Allergie“ von einer Schar fachübergreifend agierender „Allergologen “ auf hohem Niveau gemanagt werden kann, wenn man auf der anderen Seite beispielweise realisiert, dass der klassische Internist mittlerweile zum Organspezialisten mutiert ist.

Die sehr interessanten wissenschaftlichen Beiträge dieses Heftes dokumentieren eindrucksvoll, wie dies zu bewerkstelligen ist. Risiken unerwünschter Arzneimittelnebenwirkungen im Rahmen einer modernen Pharmakotherapie abzuschätzen, neue Krankheitsbilder zu erkennen, schließlich Alternativen anzubieten, stellen hier gute Beispiele dar. Und wie sieht es im Praxisalltag aus? Kann es heute noch angehen, dass Allergiker sterben aufgrund unzureichender Deklaration von Lebensmitteln? Die Kasuistiken auf Seite 572 stellen hier allenfalls die betrübliche Spitze eines Eisbergs dar.

Nach wie vor haben wir in Deutschland das Problem, dass viele Allergiekranke überhaupt nicht erkannt bzw. nur unzureichend behandelt werden. Die Folgen sind eine Chronifizierung und dadurch ausgelöst mittelfristig eine Kostensteigerung. Gibt es hier Lösungsansätze im bestehenden System? Sicher dann, wenn es den Beteiligten gelingt über den eigenen Tellerrand zu schauen. Im innerärztlichen Dialog ist aus unserer Sicht zu fordern, „Die aktuelle Diskussion um dass ein Allergiker einer Pridie geplante KBV-Reform zur märdiagnostik durch den AllLabordiagnostik ist mehr ergologen zuzuführen ist. Dieals müßig." ser hat die Weiterbildung und Kenntnis, um auch aufwändige Therapieformen wie die spezifische Immuntherapie zu initiieren. Es sollte auch unstrittig sein, dass der Allergologe hierzu notwendige diagnostische Maßnahmen, einschließlich der In-vitro-Diagnostik, bei Vorliegen entsprechender Qualifikation, durchführen darf. Insofern ist die aktuelle Diskussion um geplante KBV-Reformen zur Labordiagnostik mehr als müßig. Das geplante KBV-Kompetenzzentrum Labor ist sicher zu begrüßen, wenn es um die Etablierung von Standards in der Indikationsstellung und Qualität in der Durchführung der Labordiagnostik geht. Andererseits ist es abzulehnen, die In-vitro-Diagnostik in der allergologischen Facharztpraxis grundsätzlich in Frage zu stellen, zumal die Bestimmung der Richtig-

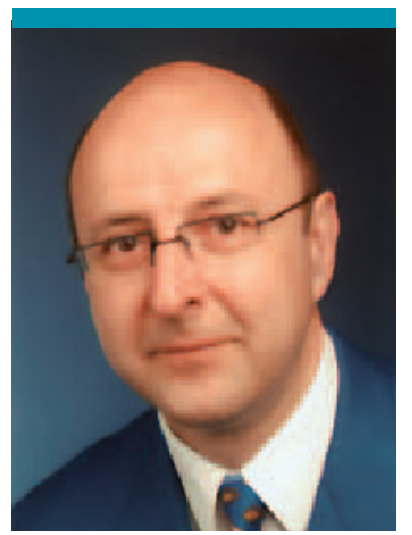

Prof. Dr. Wolfgang Czech, Praxis Dermatologie und Allergologie, VS-Villingen, Präsident des ÄDA

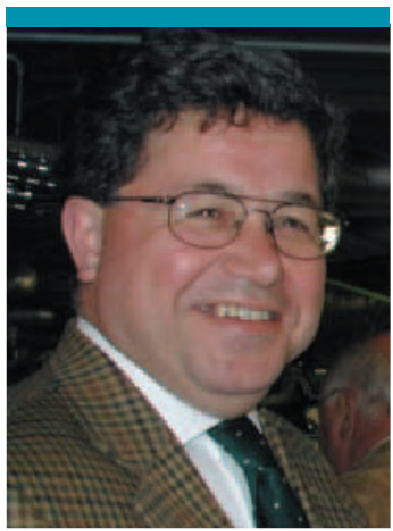

Prof. Dr. Hans F. Merk, Klinik für Dermatologie und Allergologie, Universitätsklinikum der RWTH Aachen, Vizepräsident des ÄDA keit unter Berücksichtigung des Allergenextraktes den Vergleich mit In-vivo-Testungen erfordert.

Ist Licht in Sicht? Die allergologischen Fachverbände und hier insbesondere der Ärzteverband Deutscher Allergologen als Vertretung der in der Praxis tätigen Kollegen stehen in regelmäßigem Kontakt mit der kassenärztlichen Bundesvereinigung, um den begründeten Interessen Nachdruck zu verleihen. Auch die Politik scheint das Problem allergischer Erkrankungen erkannt zu haben und etabliert aktuell unter Einbeziehung der allergologischen Fachverbände einen nationalen Aktionsplan gegen Allergien.

Die Allergologen sind für diese Herausforderungen gerüstet. In Zukunft wird ein gemeinsam durch die drei allergologischen Fachgesellschaften DGAKI, GPA und ÄDA getragener nationaler Allergiekongress durchgeführt, der neben den regionalen Veranstaltungen ein ideales Forum zur Kompetenzsicherung des Allergologen darstellen wird. Bitte machen Sie Gebrauch von diesem Angebot und planen sich die nächste Tagung in Lübeck ein (S. 550)!

Der Ärzteverband Deutscher Allergologen wünscht Ihnen und Ihrer Familie für die kommende Weihnachtszeit besinnliche Stunden sowie Glück und Gesundheit für das Neue Jahr 2007.
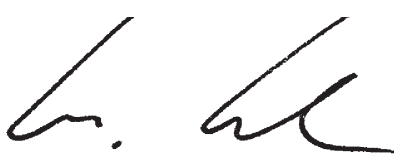

Prof. Dr. Wolfgang Czech
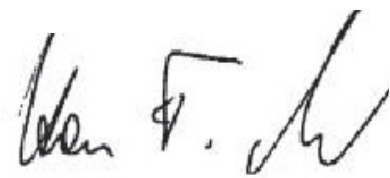

Prof. Dr. Hans F. Merk 